\title{
Accurate measurement of the specific absorption rate using a suitable adiabatic magnetothermal setup
}

\author{
Eva Natividad, ${ }^{1}$ Miguel Castro, ${ }^{1, a)}$ and Arturo Mediano ${ }^{2}$ \\ ${ }^{1}$ Instituto de Ciencia de Materiales de Aragón, CSIC-Universidad de Zaragoza, Sede Campus Río Ebro, \\ María de Luna 3, 50018 Zaragoza, Spain \\ ${ }^{2}$ Grupo de Electrónica de Potencia y Microelectrónica (GEPM), Instituto de Investigación en Ingeniería de \\ Aragón, Universidad de Zaragoza, María de Luna 3, 50018 Zaragoza, Spain
}

(Received 4 February 2008; accepted 13 February 2008; published online 5 March 2008)

\begin{abstract}
Accurate measurements of the specific absorption rate (SAR) of solids and fluids were obtained by a calorimetric method, using a special-purpose setup working under adiabatic conditions. Unlike in current nonadiabatic setups, the weak heat exchange with the surroundings allowed a straightforward determination of temperature increments, avoiding the usual initial-time approximations. The measurements performed on a commercial magnetite aqueous ferrofluid revealed a good reproducibility (4\%). Also, the measurements on a copper sample allowed comparison between experimental and theoretical values: adiabatic conditions gave SAR values only $3 \%$ higher than the theoretical ones, while the typical nonadiabatic method underestimated SAR by 21\%. (C) 2008 American Institute of Physics. [DOI: 10.1063/1.2891084]
\end{abstract}

Magnetic-fluid hyperthermia (MFH) for cancer treatment is currently attracting considerable scientific and technical work. ${ }^{1-3}$ The use of nanoscale heaters to destroy cancerous tissue allows overcoming certain problems arising from other hyperthermia therapies, ${ }^{4}$ such as damage of healthy tissue, temperature miscontrol, and use of hazardous alternating magnetic fields out of the biological range.

The heating efficiency of the fluids is quantified by the specific absorption rate (SAR), defined as the thermal power per unit mass dissipated by the active material in the presence of an alternating magnetic field. SAR highly depends on field parameters and on material properties, since different heating mechanisms can be involved. ${ }^{5,6}$ So accurate measurements are necessary for the studies on correlation between the SAR and material properties, ${ }^{7-11}$ simulations of temperature distributions in tissues or phantoms, ${ }^{12,13}$ and the optimization of hyperthermia therapies. ${ }^{1,2}$

SAR can be estimated by calorimetric methods as SAR $=(1 / m) C(\Delta T / \Delta t)$, where $m$ is the mass of the dissipating material, $C$ the heat capacity of the whole sample, and $\Delta T$ the sample temperature increase during the ac-field application interval $\Delta t$. Current SAR installations reported in literature $^{8,10,14-16}$ consist of an ac magnetic field generator, a sample space delimited by an isolating material, temperature sensors, and a data acquisition system. These setups do not provide adiabatic conditions, since heat losses (conduction, radiation, and convection) are not minimized. SAR must be estimated from the temperature-versus-time exponential curve, ${ }^{16}$ according to the expression $\mathrm{SAR}=C \beta / m$, where $\beta=\left.(d T / d t)\right|_{t \rightarrow 0}$ is the initial slope. This procedure can lead to unknown errors in the determination of $\beta$ and, therefore, to incorrect SAR values, if the initial thermal losses are not negligible, or if there is not a homogeneous temperature distribution across the sample, facts that are not easy to infer in practice.

${ }^{\text {a) }}$ Author to whom correspondence should be addressed. Electronic mail: mcastro@unizar.es.
In this letter, we report accurate SAR measurements using an adiabatic magnetothermal setup, ${ }^{17}$ in which the sample undergoes only a weak net heat exchange with the surroundings, overcoming the previous limitations. In such conditions, the generated heat can be considered to be entirely invested in the sample temperature raise, allowing direct measurement of $\Delta T$ and providing more precise SAR values.

The adiabatic magnetothermal setup (Fig. 1) allows measuring for both solid and liquid samples. For the latter, a quartz sample holder sealed with a vacuum-proof solventfree adhesive is suitable. The sample is hanged by thermal isolating threads from the adiabatic shield, which surrounds the sample. For the realization of adiabatic conditions, this shield must be continuously controlled to the same temperature of the sample. In adiabatic calorimetry, good temperature control is achieved with metallic shields. ${ }^{18}$ However, in our setup, this shield is made of alumina, due to the impossibility of using metallic parts in the vicinity of the sample, since these parts would rapidly heat up due to Foucault currents, in the presence of the ac magnetic fields suitable for

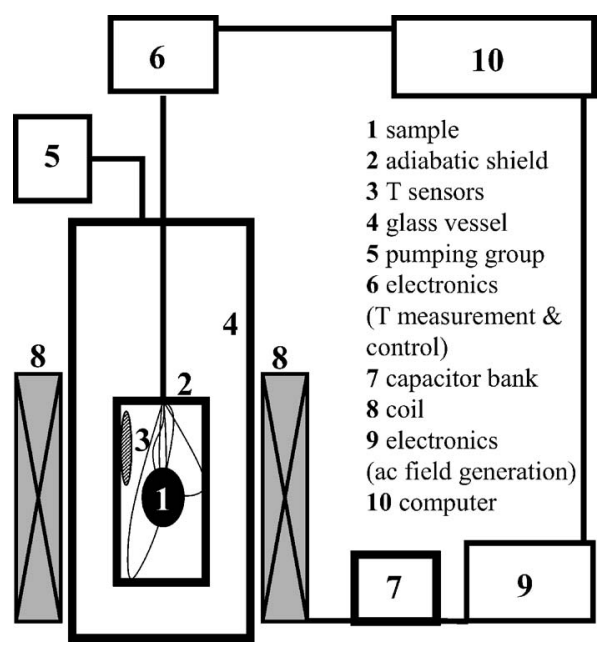

FIG. 1. Scheme of the adiabatic magnetothermal setup. 


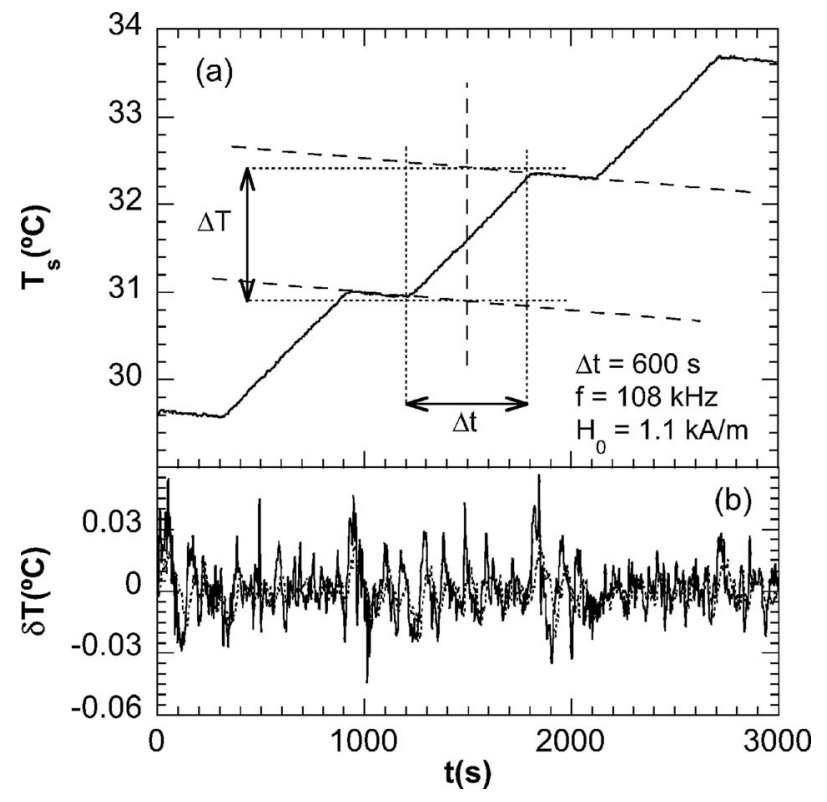

FIG. 2. Three consecutive heating steps of the ferrofluid sample. (a) Temperature of the sample. (b) Error signals of the temperature control, $\delta T_{1}$ (solid line) and $\delta T_{2}$ (dashed line).

$\mathrm{MFH}$, and also would alter the uniformity of such magnetic fields. In more detail, the shield consists of a cylinder with a lid, each provided with a meander-shaped nickel-alloy heater on its outer face. These meanders $(\sim 1.5 \mu \mathrm{m}$ thick) are thin enough to undergo negligible field-induced self-heating as well as to produce adequate heating power for temperature control when fed by an external current. The temperature sensors are three nonferromagnetic thermocouples and a resistance thermometer. One thermocouple measures the temperature of the sample $T_{s}$. The other two, in differential configuration, provide the cylinder-sample $\left(\delta T_{1}\right)$ and lidcylinder $\left(\delta T_{2}\right)$ error signals for the proportional-integralderivative (PID) temperature controllers. For these three signals, shielding and filtering of electromagnetic interferences are essential. Finally, the thermometer provides accurate absolute temperature values in the absence of the ac magnetic field. The sample, shield, and sensors are enclosed in a vacuum environment by means of a glass vessel. A turbomolecular pumping group provides final pressures of about $8 \times 10^{-5}$ mbar.

The alternating magnetic field is generated by a series resonant circuit (a capacitor bank and a coil) excited by a power amplifier working in high efficiency class-D conditions. The coil is placed outside the glass vessel, so that the eventual heating at high field amplitudes does not interfere in the adiabatic control. ac currents in the frequency range of $f=50-500 \mathrm{kHz}$ feed the coil and field amplitudes $H_{0}$, up to $4 \mathrm{kA} / \mathrm{m}$ can be reached. Field uniformity is about $99 \%$ within a cylindrical volume of $50 \mathrm{~cm}^{3}$ at the coil center as predicted through the use of electromagnetic computer simulation.

Measurements take place according to the pulse heating method used in traditional adiabatic calorimetry. ${ }^{18}$ With continuous temperature control of the shield, the sample temperature is registered before, during, and after field application. Consecutive steps (Fig. 2) can be performed in a narrow temperature range. Corrections of the small thermal losses are performed by linear fitting of temperature drifts in both Downloaded 19 Feb 2010 to 161.111 .180 .191 . Redistribution subje
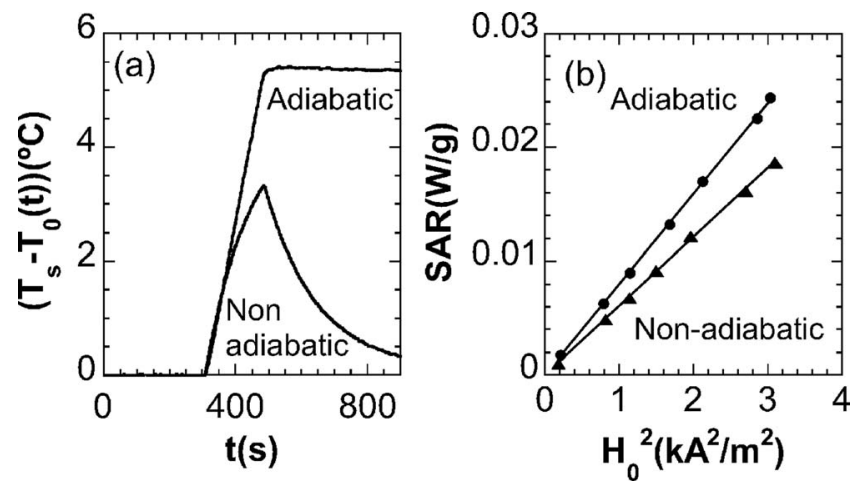

FIG. 3. (a) Adiabatic vs nonadiabatic heating steps of the copper sample $\left(f=108 \mathrm{kHz}, H_{0}=1.3\right.$, and $\left.1.4 \mathrm{kA} / \mathrm{m}\right)$. To help in the comparison, $T_{s}$ $-T_{0}(t)$ was displayed instead of $T_{s}$ with $T_{0}(t)$ standing for the temperature linear trend before field application. (b) Dependence of adiabatic and nonadiabatic SAR values with $H_{0}{ }^{2}$.

equilibrium processes. $\Delta T$ is obtained by the extrapolation of both drift rates toward the midpoint of $\Delta t$. Eventually, the setup can also be used in nonadiabatic conditions only by disabling the temperature control and the pump. In this case, cooling down to room temperature is required between steps.

Adiabatic measurements over a ferrofluid were performed with a commercial product from Chemicell $\mathrm{GmbH}$, namely, the fluid MAG-UC/A. It is an aqueous dispersion of magnetite nanoparticles with average hydrodynamic diameter, concentration, and density of $50 \mathrm{~nm}, 100 \mathrm{mg} / \mathrm{ml}$, and $1.25 \mathrm{~g} / \mathrm{cm}^{3}$, respectively, according to supplier specifications. The sample consisted of $0.3387 \mathrm{~g}$ of ferrofluid (0.0271 g of magnetite) in a quartz sample holder of $1.1561 \mathrm{~g}$, sealed with $0.045 \mathrm{~g}$ of adhesive, with a total $C$ of $2.267 \mathrm{~J} /{ }^{\circ} \mathrm{C}$, estimated from literature values.

The linear trends during equilibrium due to the residual heat exchange [Fig. 2(a)] present slopes of $2 \times 10^{-4}{ }^{\circ} \mathrm{C} / \mathrm{s}$, and the PID controls [Fig. 2(b)] keep temperature error signals below $\pm 0.03{ }^{\circ} \mathrm{C}$, revealing acceptable adiabatic conditions. The SAR value obtained by averaging seven heating steps in the same conditions is $0.217 \pm 0.009 \mathrm{~W} / \mathrm{g}$, pointing good reproducibility $(4 \%)$.

In order to evaluate the accuracy and give an estimation of the improvements obtained with the adiabatic method, a copper cylinder with length, radius, and mass of $L=19.85 \mathrm{~mm}, R=1.30 \mathrm{~mm}$, and $m=0.8956 \mathrm{~g}$, respectively, was used as a standard. The theoretical analytical expression for the heating power dissipated due to Foucault currents by a metallic semi-infinite cylinder in a uniform axial alternating magnetic field, provided that the skin depth $\delta=(\pi f \mu \sigma)^{-1 / 2}$ is much smaller than $R$, is $P=H_{0}{ }^{2} \pi R L /(\sigma \delta),{ }^{19}$ where $\sigma$ is the material electrical conductivity and $\mu$ the magnetic permeability, and then $\mathrm{SAR}=P / m$.

Nonadiabatic (exponential trend) and adiabatic (linear trend) measurements were carried out [Fig. 3(a)]. For the nonadiabatic case, $\beta$ was estimated by linear fitting the sample temperature variation with time, starting at the onset of the magnetic field application, and considering time intervals of $60 \mathrm{~s}$, which gave maximum slopes. The $C$ value was $0.384 \mathrm{~J} /{ }^{\circ} \mathrm{C}$. The dependence of the SAR values on $H_{0}{ }^{2}$ is linear [Fig. 3(b)] as expected, with slopes of 0.00795 (adiabatic) and $0.00607 \mathrm{~W} \mathrm{~m}^{2} / \mathrm{g} \mathrm{kA}^{2}$ (nonadiabatic). Considering $\sigma_{\mathrm{Cu}}=5.88 \times 10^{7} \Omega^{-1} \mathrm{~m}^{-1}$, the analytical expression derives a slope of $0.00771 \mathrm{~W} \mathrm{~m}^{2} / \mathrm{g} \mathrm{kA}^{2}$; that is, the adiabatic SAR to AlP license or copyright; see http://apl.aip.org/apl/copyright.jsp 
values only differ by $3 \%$ from theoretical ones, while the nonadiabatic determination underestimates the theoretical SAR by $21 \%$. These results have allowed, on the one hand, to evaluate the accuracy in SAR determination by calorimetric methods and, on the other hand, to point the improvement of the adiabatic over the nonadiabatic method, by using the same setup.

As final conclusions, accurate and reproducible SAR measurements of solid and liquid samples were obtained by means of a specifically designed adiabatic setup, which represents a great improvement with respect to the current nonadiabatic determination.

Financial support from the Spanish MEC (Project No. MAT2004-03395-C02-02) is acknowledged. Eva Natividad thanks the Spanish CSIC for her I3P Contract (European Social Fund).

${ }^{1}$ M. Johannsen, U. Gneueckow, B. Thiesen, K. Taymoorian, C. H. Cho, N. Waldofner, R. Scholz, A. Jordan, S. A. Loening, and P. Wust, Eur. Urol. 52, 1653 (2007)

${ }^{2}$ M. Kettering, J. Winter, M. Zeisberger, S. Bremer-Streck, H. Oehring, C. Bergemann, C. Alexiou, R. Hergt, K. J. Halbhuber, W. A. Kaiser, and I. Hilger, Nanotechnology 18, 175101 (2007).

${ }^{3}$ Q. Zeng, I. Baker, J. A. Loudis, Y. Liao, P. J. Hoopes, and J. B. Weaver, Appl. Phys. Lett. 90, 233112 (2007).

${ }^{4}$ P. Wust, B. Hildebrandt, G. Sreenivasa, B. Rau, J. Gellermann, H. Riess,
R. Felix, and P. M. Schlag, Lancet Oncology 3, 487 (2002).

${ }^{5}$ R. Hergt and W. Andrä, in Magnetism in Medicine, edited by W. Andrä and H. Nowak (Wiley-VCH, Weinheim, 2007), p. 550, and references therein.

${ }^{6}$ R. E. Rosensweig, J. Magn. Magn. Mater. 252, 370 (2002).

${ }^{7}$ S. Dutz, R. Hergt, J. Murbe, R. Muller, M. Zeisberger, W. Andrä, J. Topfer, and M. E. Bellemann, J. Magn. Magn. Mater. 308, 305 (2007).

${ }^{8}$ K. Okawa, M. Sekine, M. Maeda, M. Tada, M. Abe, N. Matsushita, K. Nishio, and H. Handa, J. Appl. Phys. 99, 08H102 (2006).

${ }^{9}$ S. Bae, S. W. Lee, and Y. Takemura, Appl. Phys. Lett. 89, 252503 (2006).

${ }^{10}$ J. P. Fortin, C. Wilhelm, J. Servais, C. Menager, J. C. Bacri, and F. Gazeau, J. Am. Chem. Soc. 129, 2628 (2007).

${ }^{11}$ A. S. Eggeman, S. A. Majetich, D. Farrell, and Q. A. Pankhurst, IEEE Trans. Magn. 43, 2451 (2007).

${ }^{12}$ R. Xu, Y. Zhang, M. Ma, J. Xia, J. Liu, Q. Guo, and N. Gu, IEEE Trans. Magn. 43, 1078 (2007).

${ }^{13}$ N. Tsafnat, G. Tsafnat, T. D. Lambert, and S. K. Jones, Phys. Med. Biol. 50, 2937 (2005).

${ }^{14}$ G. Glockl, R. Hergt, M. Zeisberger, S. Dutz, S. Nagel, and W. Weitschies, J. Phys.: Condens. Matter 18, S2935 (2006).

${ }^{15}$ S. Bae, S. W. Lee, Y. Takemura, E. Yamashita, J. Kunisaki, S. Zurn, and C. S. Kim, IEEE Trans. Magn. 42, 3566 (2006).

${ }^{16}$ I. Hilger, K. Fruhauf, W. Andrä, R. Hiergeist, R. Hergt, and W. A. Kaiser, Acad. Radiol. 9, 198 (2002).

${ }^{17}$ M. Castro, A. Mediano, E. Natividad, and F. Palacio, "Equipo adiabático para medida del coeficiente de absorción específico de un material sometido a un campo magnético alterno," Spanish Patent Application No. P200703426 (pending).

${ }^{18}$ E. Gmelin, Thermochim. Acta 29, 1 (1979).

${ }^{19}$ M. T. Thompson, IEEE Trans. Magn. 34, 3755 (1998). 